\section{Reports of Societies}

\section{SWINE ERYSIPELAS INFECTION IN MAN AND ANIMALS}

A meeting of the Section of Comparative Medicine of the Royal Society of Medicine was held on Feb. 18, when the subject for discussion was swine erysipelas infection (Erysipelothrix rhusiopathiae) in man and animals.

\section{Veterinary Problems}

Mr. A. W. Gledhill said that this disease of pigs was almost world-wide, though it varied in seriousness from country to country and even from region to region. In Britain its prevalence had been most marked in East Anglia. Susceptibility to infection was not confined to pigs and the disease occurred commonly in birds of most species. The organism was also a common cause of arthritis in lambs, and it had been described as causing endocarditis in horses. There was a good deal of evidence to indicate that it might lead a saprophytic existence. In areas where the disease appeared subclinical infections were common. Pigs between the ages of three months and one year were most susceptible. No doubt sucking-pigs received protective antibodies from the sow, and pigs over one year might have acquired sufficient immunity to resist infection. Lowered host resistance might play a dominant part in determining the conditions necessary for the onset of a severe outbreak. There were three forms of swine erysipelas: mild, acute septicaemic, and chronic. Immune serum acted by neutralizing the toxic effect of the organisms rather than by promoting their destruction. As curative agents the sulphonamides were not of value; the organism was sensitive to penicillin in vitro, and to a less extent to streptomycin.

\section{Human Infections}

Dr. Mary Barber, of the British Postgraduate Medical School, gave a survey of the history and literature of infection in man. Compared with swine, she said, man was relatively immune to infection with this organism. Three types of human infection occurred, namely, cutaneous, intestinal, and generalized. The first was by far the commonest, and to this she devoted most of her remarks. A case of cutaneous infection of this nature was described in the British Medical Journal as long ago as 1870. It was the case of a foreman in a sheepskin bootshop on whose hands lesions developed; the recurrence of the disease was ultimately prevented by the wearing of gloves. [This patient, a man aged 28, was in the care of Dr. Tilbury Fox at University College Hospital, who described the case in our issue of Feb. 5, 1870, at page 132.] In 1873 a worker at St. Bartholomew's Hospital reported 16 cases of a similar condition, in several of which the lesion appeared to have originated in a scratch from a bone while handling meat. The disease was established as a clinical entity in the late 'eighties, and its causal relation with swine erysipelas was worked out. The condition was met with fairly frequently in a large skin clinic. Infection occurred mainly by accidental transmission among those who were brought into contact with pig infection, such as cooks, kitchen workers, and butchers, also among people who handled fish. Nearly always there was a history of injury. The period of incubation was from one to five days. The disease was confined almost exclusively to the hands, rarely spreading above the wrist, but there were a few cases in which the lesion was on the soles of the feet or on the face or neck. The patient complained of burning, itching, and pain. The lesions were of a purple-red colour, with definite margins. Glandular enlargement was very rare, and suppuration was never recorded. Fever and constitutional symptoms were slight and transitory. The lesions might disappear spontaneously without desquamation, but recurrence was not infrequent.

Serum had been the usual method of treatment, but serum sickness was very commonly produced and was more trying than the infection itself. Sulphonamides had been used with doubtful effect, though there were reports of cure. A few cases had been treated with penicillin, and she thought there was evidence that this had some effect. In one case, within two days of starting treatment with penicillin there was almost nothing to be seen on the hand.

Dr. Barber showed figures illustrating the experiences of various investigators and also described some cases of generalized. infection. One of these was a case of septicaemia associated with endocarditis. The patient died a month after admission to hospital, the whole course of the disease having lasted four months. At necropsy the heart size was found to be nearly twice normal, and vegetations adhered to the anterior cusp of the mitral valve. One case was recorded in whict infection was via the alimentary tract. In this case the patien developed malaise, anorexia, and fever, with the eruption of red spots on the trunk and limbs, later a painful swelling of the knee-joint and severe anaemia and leucopenia. He improved after the administration of immune serum but relapsed and died; no post-mortem examination was carried out.

\section{Effectiveness of Chemotherapy}

Mr. Malcolm Woodbine, of the Wellcome Laboratories 4 Beckenham, reported some observations on the effectivenes: of the new chemotherapeutic agent upon Erysipelothrix rhusiopathiae infections in mice. The now substantial literature on the ineffectiveness of sulphonamide compounds owing to their mode of action, as in the case of sulphathiazole, sulphadiazine. sulphapyrazine, sulphanilylguanidine, phthalylsulphathiazoles and sulphetrone, had been confirmed in vitro and in vivo. but it was of interest, to note that "marfanil," or benzy]-. amine-4-sulphonamide, which owed its activity to a different mechanism, was active in vitro.

Results with penicillin indicated that very large doses were necessary. The optimal dose was 1,000 units twice daily per mouse (20 g.) for ten days or more, and this might be compared with the total dosage of the order of 30 units per 20-g mouse for complete protection in streptococcal, staphylococcal, and pneumococcal infections. The necessity of parenteral, administration of relatively large doses appeared to preclude the use of penicillin in swine erysipelas. Recent work in the United States, in which turkeys had been used and doses of 20,000 units once daily over four days had been given, showed a reduction of mortality from $100 \%$ in controls to $10 \%$. Pigeons had been cured with penicillin, and the use of penicillin successfully in man had been recorded not only by Dr. Barber and her colleagues (1946), but by Hodgson (1945), Jennin (1946), and Stiles (1947).

The reported activity of streptomycin in vitro against this organism encouraged the laboratories at Beckenham to examine this antibiotic when supplies became available. Streptomycin was active in vitro and in vivo in mice, but less active than penicillin weight for weight. There was, however, a synergic action in mouse infections when penicillin and streptomycin were administered together in equal doses, and this might be of some potential value. Aerosporin showed little activity in vitro and no activity at all in infections in mice. The related antibiotic polymyxin was reported to be inactive in vitro. II appeared that a still more effective chemotherapeutic agent. suitable for oral administration, was required, particularly in the veterinary field.

In some further discussion a veterinary surgeon said that fatal cases in man were very rare, but a case came under his. notice in Colombo, in a laboratory assistant, whose illness was first described as malaria, from which he had previously, suffered. But it did not respond to quinine, and he became steadily worse. The symptoms were intermittent fever, weakness, and loss of weight. Typhoid, brucellosis, and malaria were excluded. The patient died, and at necropsy nothing, characteristic was found except some enlargement of the spleen, from which, however, the organism was cultured.

Dr. BARBER said that in describing man as relatively immune she meant that it was rare to see the disease in a severe form. The lesions were slight and healed up quickly, so that the cases did not come into the hands of the bacteriologist.

The PREsident said that swine erysipelas had always been a serious problem in veterinary medicine. Before the war a good deal of vaccination was done, and up to a point was satisfactory. During the war, with the decline in pig population, the disease tended to die out, but with the building up of herds there might 
be a recrudescence. He wondered whether the susceptibility of man to this disease might not be much higher than was commonly thought.

\section{CLINICAL PATHOLOGY \\ LETTSOMIAN LECTURE}

The first of three Lettsomian Lectures on "Clinical Pathology in Relation to Medical Practice" was delivered by Dr. CuTHBERT E. DUKES before the Medical Society of London on Feb. 16. The President of the Society, Mr. W. E. TANNER, was in the chair.

Dr. Dukes began by saying that one reason why clinical pathology deserved consideration at the present time was because of the intention of the Health Service Act to extend laboratory facilities to the whole of the community. Up to the present, in most districts, only hospital patients had enjoyed the benefit of a free comprehensive service. Now the door of the laboratory was to be opened to all-a prospect which the clinical pathologist might regard with some satisfaction not unmixed with apprehension. Increased facilities for clinical pathology would be welcomed by the profession, especially by general practitioners, but a service of this sort could not be started in a few weeks. Laboratories had to be built and equipped, and pathologists and technicians had to be trained.

\section{Definition}

Clinical pathology might be defined as the application of pathology and pathological method in clinical practice. Much of the diagnosis and treatment of disease had come to depend on team work, and an essential member of the team was the pathologist. The mastery of laboratory technique was neither easily nor quickly acquired, and it was in order to meet the need for co-ordination between clinical medicine and laboratory science that the clinical pathologist was evolved. The difference between academic and clinical pathology was no greater than between pure and applied chemistry or pure and applied mathematics. Academic pathology was a pure science, clinical pathology the application of science to the diagnosis and treatment of disease in the individual patient.

The development of clinical pathology in the past had gone through three phases. When hospitals first recognized the need for a pathological department it was usual for the physician with a liking for the microscope or for chemistry to be asked to "look after" pathology. The next phase of development was for the post of pathologist to be regarded as a stepping stone to the staff. The third stage, the result of the rapid extension of the subject during the last twenty or thirty years, was that in universities and medical schools the pathologists continued to specialize still further, thereby creating difficulties in the application of pathology to clinical medicine. A single pathological specimen sent to a departmentalized laboratory tended to be passed from one department to another. This piecemeal analysis, though occasionally necessary in obscure cases, was not desirable in the ordinary case. A specimen such as cerebrospinal fluid should be examined as a whole by a pathologist who understood something of the clinical side of the case.

In the smaller provincial hospitals the evolution of clinical pathology proceeded along different lines. Many curious devices were adopted by some voluntary hospitals to raise funds for laboratories. Some turned themselves into trading concerns and offered to examine all specimens sent in by general practitioners. Pathology had often been described as a science ancillary to medicine, but some pathologists came to think of themselves not as handmaids but as household drudges. Until recently very few facilities existed outside the hospitals. Diagnostic laboratories established by public health authorities, research institutions, and commercial firms catered only for certain sections. One of the defects of National Health Insurance was the lack of facilities for clinical pathology. The Act made provision for any drug, remedy, or therapeutic agent to be prescribed by the insurance practitioner, but it did not enable him to find out whether the agent prescribed was really needed. In pernicious anaemia he was allowed to prescribe liver extract, but he had no facilities for taking blood counts.

\section{To-day and To-morrow}

In the Emergency Medical Service in 1939 provision was at once made for laboratory diagnosis and clinical pathology as an integral part of wartime hospital organization. At the moment facilities for pathological services varied greatly in different parts of the country, and pathologists themselves were scattered about in a haphazard fashion. The survey recently undertaken by the Ministry of Health drew attention to the unequal distribution of facilities for pathological tests and to lack of co-ordination and failure to make use of the facilities which existed.

It was difficult to foresee how clinical pathology would develop in the future. The only certain thing about the future, as Lord Keynes said, was that it would be different from the past. Plans were in preparation for a considerable extension of hospital and public health laboratory work, but were handicapped by shortage of personnel and equipment. On the appointed day hospital pathological laboratories would come under the control of the Regional Hospital Boards. It was not yet clear how the hospital services would be co-ordinated with those at present organized separately by the public health services. The investigation and control of epidemics was obviously the task of the public health epidemiologist, but the diagnosis in the case of an individual patient might depend on tests carried out by a hospital pathologist. It was anticipated that in the future the public health service would provide free diagnostic facilities for all infectious diseases, together with central reference laboratories. It remained to be seen whether it would be best to run the two services separately or in combination.

The efficiency of the pathological services provided under the new Act would depend largely on the supply of well-trained clinical pathologists, and on the spirit in which they did their work. Up to the present clinical pathologists holding teaching appointments or attached to voluntary hospitals had enjoyed a large measure of independence. They had been answerable to committees or governing bodies, but these had very seldom interfered with arrangements made for running the laboratories. Under the new Act the pathologist might not enjoy as much freedom as in the past, but he hoped to gain other advantages in return. They would all have to be on their guard lest the new control resulted in a shifting of attention from the patient to some other objective. The art of managing and manipulating other people had now been carried to an extraordinary level of efficiency, but the more they exercised social control over their fellows the more they tended to lose sight of the real needs of the individual.

\section{Training the Clinical Pathologist}

The clinical pathologist should be well trained clinically a: well as technically. It had been recommended by the Consulting Pathologists Group of the B.M.A. that future entrants should not be recognized as specialists in clinical pathology until after five years' postgraduate experience and the attainment of a higher qualification, and that no assistant should be admitted to the staff of a department of clinical pathology until he or she had done at least one year in a house appointment. The sound way of acquiring training was in a general hospital under a good all-round clinical pathologist. In universities and colleges where specialization had led to the formation of a number of separate departments there was a tendency for the budding pathologist to bud out too luxuriously in one direction to the detriment of others.

Certain personal characteristics were necessary for success in any profession. For the clinical pathologist one of the most important qualifications was the capacity for working with other people, especially medical and surgical colleagues. fellow pathologists, and laboratory technicians. Team work was essential for good clinical pathology. Next in importance was a capacity to direct the activities of others. The chief pathologist in a laboratory was not unlike the conductor of an orchestra. Ideally the conductor should be able to play every instrument ; but that was too much to ask, and all that was essential was that he should know each instrument's capabilities and limitations. So with the pathologist and the various laboratory procedures. 
It was often erroneously assumed that personal relationships were of less importance in pathology than in other branches of medicine. The extent to which a pathologist entered into any sort of personal relationship with patients varied greatly according to the nature of his work, but whether he met the patient personally or only a bit of the patient, the existence of the patient as a living individual must never be overlooked. Their patients were not the objects but the subjects of their skill. The public in general adopted a foolish attitude to pathologists, just as they did to all scientists. Patients either took the view that nothing of value would come from the pathologist's activities, or else they looked with childlike faith upon laboratory mysteries as their only hope.

The personal relationship of the pathologist and the laboratory technicians affected the working of the laboratory much more than an outsider might suppose. The whole atmosphere of the laboratory might easily be poisoned by one discontented assistant. Trained laboratory assistants should always be made to feel that they were in responsible positions.

Finally, with regard to the relationship of the pathologist to his professional colleagues, doctors in general differed to a remarkable degree in their attitude to pathological services and in the confidence they reposed in a pathologist's report. Some referred to the laboratory as if it were an inspired oracle; others suppressed all laboratory emanations as irrelevant or misleading. The great majority resorted to the laboratory only for an occasional Wassermann or pregnancy test. It would be a disaster, of course, if laboratory tests led to the neglect of the clinical examination. They should be regarded not as a substitute for but only as an extension of the clinical findings.

\section{THE FILMSTRIP IN MEDICAL TEACHING}

A joint meeting of the Royal Society of Medicine and the Scientific Film Association was held, under the chairmanship of Mr. ZACHARY COPE, on Feb. 12 to discuss medical filmstrips. A filmstrip is a length of cine film on which a series of pictures is printed in a desired sequence and projected on a screen, very much in the same way as a series of lantern slides. The pictures may be reproductions of printed material, such as illustrations or book text, original drawings or paintings, and, of course, photographs and radiographs.

Dr. R. M. TATTERSALl said that on seeing filmstrip for the first time a year ago he was so impressed by its possibilities in medical teaching that he set to work at the medical school at Leeds to apply it to instruction in such techniques as lumbar puncture, the taking of the blood sedimentation rate, catheterization, and other simple procedures. In making the filmstrips they endeavoured to be as elementary and detailed as possible, the first frame, perhaps, reproducing the initial request for the procedure, and the last of the thirty or more the written-up case-notes. They had no special department of medical photography at Leeds, but one of the registrars had a Leica camera, and with this the negatives had been obtained, the processing being given out to a trade house. The magazine capacity of the Leica imposed the limitation of about 34 frames in one strip. In deciding on what was to be shown it had to be borne in mind that no two people carried out, for example, lumbar puncture in exactly the same way. Therefore the filmstrips were produced on a committee basis, three or four people sitting down together, arguing out their differences, and agreeing on the points to be emphasized in the successive pictures. Title and captions were, also decided, and, in addition to the photographs in sequence of the actual procedure, charts, diagrams, pictures of the instruments to be used, and illustrations from anatomical specimens might be included. One or more frames might be used to remind the student pictorially of the importance of observing absolute cleanliness. The last few frames of the strip might illustrate the labelling of the specimen bottles, the completion of the case-notes, and perhaps the medical uses of the procedure. At least five people were required for the making of the filmstrip, namely, the member of the staff who carried out the procedure on the patient, the photographer, someone to attend to the lighting arrangements, someone else to follow what in cinematograph parlance was called the script, and finally the patient himself. who must be co-operative. It was as well to have a rehearsal. In projecting the filmstrip it was first taken slowly through the lantern, then quickly to give the illusion of continuity to the photographs of the actual procedure. A group of students should first be shown the filmstrip, then see the actual procedure, and after that they should observe the filmstrip again.

Dr. BRIAN STANFORD reminded the meeting that $35-\mathrm{mm}$. film was inflammable, whereas $16-\mathrm{mm}$. substandard film was safe for projection in places not specially protected. A positive filmstrip could be made straight from the camera by reversal. but that was not to be recommended; it was better to make negatives and print from them. If diagrams only were wanted they could be drawn in white ink on black paper, photographed and developed, and projected on the screen as black on white. and this was perfectly satisfactory for many purposes but of course was applicable only to line drawings or printed matter

Various technical points were brought out in further discussion, such as density, tone range, and screen brightness. It was stated that the average cost of a filmstrip was about 12s. 6d At the close of the meeting a number of filmstrips illustrating, medical subjects were shown.

\section{THE LESSONS OF WAR SURGERY}

At a meeting of the Manchester Medical Society on Feb. + Sir Heneage OgiLvie read a paper on "The Lessons of the War for Civil Surgery."

During the recent war much was learned about transport. organization of forward surgery, segregation of special cases. and methods of wound treatment applicable only to the forces of a victorious alliance in the concluding stages of a campaign. The outstanding problems of war surgery throughout the ages were those of shock and infection. These problems had not been solved, but their treatment had been brought to a vers satisfactory level, the first by the elaboration of methods of storing blood and plasma and the organization of a resuscitation service, the second by chemotherapy. The same methods were available in civil practice, and would be applied so much the better if the simple outlook and sharp distinction between theory and practice that characterized Service units in the field were translated to the treatment of industrial accidents. The general lessons of wound treatment, excision, drainage, twostage closure, and, later, of skin cover all had their civil applications.

In addition to progress in the treatment of these fundamental problems of trauma, the level of surgical technique advancede to a remarkable extent, aided by the development of resuscitation and chemotherapy, but above all by the parallel advances in anaesthesia. The most striking example of technical advance was the change from the conservative attitude of war surgeons towards chest wounds in 1941 to the radical thoracic surgery of 1945. The freedom of the open thorax was the outstanding new feature in post-war surgery. Whereas formerly only extrapleural approaches to the heart were considered, and littler was undertaken beyond embolectomy and pericardiolysis, to-day incisions were chosen to give the most direct approach to the chamber or vessel concerned. Removal of the lung or oesophagus, daring feats before the war, was now undertaken every week. Total gastrectomy by the abdominal route was a diffi. cult and dangerous operation; now the trans-diaphragmatic approach had made the operation reasonably straightforward. The war surgery of head injuries and vascular lesions had also* left its legacy of advance to the planned surgery of peace.

In more general terms, war brought to all Service surgeons periods of rest and opportunities for discussion in which many traditional ideas were weighed and found wanting. In particulai the principles of rest and rehabilitation had been radically revised for the good of surgery and the surgical patient.

The fifth dinner meeting of the 1948 session of the Chelsea Clinicat Society was held on Feb. 10 at the South Kensington Hotel, with the president, Dr. Neil Maclay, in the chair. There was a large attendance of members and visitors to hear a discussion on "Some Difficulties in the Science of Crime Detection " opened by Dr. Keith Simpson. The discussion was continued by Dr. Donald Teare, Mr. A. Le Mesurier, Dr. Sundell, Mr. Ivor Back, Dr. MacManus. and Dr. Clive Shields. 